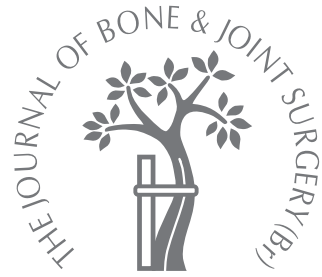

P. E. Murtha, M. A. Hafez, B. Jaramaz, A. M. DiGioia III

From Institute for Computer Assisted Orthopaedic Surgery, The Western Pennsylvania Hospital, Pittsburgh, Pennsylvania, USA

In P. E. Murtha, PhD, Research Scientist

M. A. Hafez, FRCSEd, MD, Orthopaedic Surgeon

B. Jaramaz, PhD, Director Institute for Computer Assisted Orthopaedic Surgery The Western Pennsylvania Hospital, Suite 242, Mellon Pavillion, 4815 Liberty Avenue, Pittsburgh, Pennsylvania 15224, USA.

- A. M. DiGioia III, MD, Orthopaedic Surgeon

University of Pittsburgh Schoo

of Medicine and the

Orthopaedic Program Magee Women's Hospital, 300 Market Street, Suite 1601, Pittsburgh, Pennsylvania 15213, USA.

Correspondence should be sent to Dr P. E. Murtha; e-mail: pmurtha@icaos.org

(C)2008 British Editorial Society of Bone and Joint Surgery doi:10.1302/0301-620X.90B3. $19548 \$ 2.00$

$J$ Bone Joint Surg $[B r]$ 2008;90-B:308-13.

Received 12 April 2007;

Accepted after revision 13 November 2007

\title{
Variations in acetabular anatomy with reference to total hip replacement
}

\begin{abstract}
Three-dimensional surface models of the normal hemipelvis derived from volumetric CT data on 42 patients were used to determine the radius, depth and orientation of the native acetabulum. A sphere fitted to the lunate surface and a plane matched to the acetabular rim were used to calculate the radius, depth and anatomical orientation of the acetabulum. For the 22 females the mean acetabular abduction, anteversion, radius and normalised depth were $57.1^{\circ}\left(50.7^{\circ}\right.$ to $\left.66.8^{\circ}\right), 24.1^{\circ}\left(14.0^{\circ}\right.$ to $\left.33.3^{\circ}\right), 25 \mathrm{~mm}(21.7$ to 30.3$)$ and $0.79 \mathrm{~mm}(0.56$ to $1.04)$, respectively. The same parameters for the 20 males were $55.5^{\circ}\left(47.7^{\circ}\right.$ to $\left.65.9^{\circ}\right), 19.3^{\circ}$ $\left(8.5^{\circ}\right.$ to $\left.32.3^{\circ}\right), 26.7 \mathrm{~mm}(24.5$ to 28.7$)$ and $0.85 \mathrm{~mm}(0.65$ to 0.99$)$, respectively.

The orientation of the native acetabulum did not match the safe zone for acetabular component placement described by Lewinnek. During total hip replacement surgeons should be aware that the average abduction angle of the native acetabulum exceeds that of the safe zone angle. If the concept of the safe zone angle is followed, abduction of the acetabular component should be less than the abduction of the native acetabulum by approximately $10^{\circ}$.
\end{abstract}

Dislocation rates following total hip replacement (THR) range from $1 \%$ to $4 \% .^{1-4}$ Technical factors, as distinct from patient characteristics, implicated in increasing the risk of dislocation include malpositioning or inappropriate sizing of the components, limited experience of the surgeon, and pelvic malrotation during surgery. ${ }^{5}$ In particular, the positioning of the acetabular component influences the risk of dislocation, impingement, polyethylene wear and the overall stability of the THR. ${ }^{6,7}$

Based on a radiological analysis of the observed dislocation rates in a series of 300 THRs, Lewinnek et al $^{8}$ suggested a safe zone for the orientation of the acetabular component, recommending a lateral opening of $40^{\circ}$ (SD $10)$ and anteversion of $15^{\circ}$ (SD 10) relative to the anterior pelvic plane. This early definition of a safe zone has become the de facto standard for comparison even though the ranges for acetabular component inclination and anteversion remain controversial. ${ }^{7}$ The concept of combined component position was recently suggested by Barsoum et al. ${ }^{9}$ Although many surgeons apply the safe zone to guide acetabular component orientation during THR, others use the native orientation of the acetabulum or the transverse acetabular ligament ${ }^{10}$ as a guide. ${ }^{6}$ Even though native acetabular anat- omy, as described by its radius, depth and orientation, is important to hip reconstruction, prosthetic design and the restoration of hip mechanics, very little is reported on it in the literature.

In this study we hypothesise that the native acetabulum is a good guide for placement of a prosthetic acetabular component. To test this assumption we have examined the anatomy of the acetabulum using 3D surface models of the pelvis. The parameters we studied included the acetabular radius, depth and anatomical orientation in terms of abduction and version.

\section{Materials and Methods}

The data were derived from a clinical trial of image-based, navigated THRs conducted with institutional review board approval and informed consent from the patients. These data have formed the basis of the statistical atlases of healthy pelves reported elsewhere. ${ }^{11}$ The data include the volumetric CT dataset, anonymised patient demographics, and clinical information for each patient. The clinical trial extended from 1997 to 2004 at two different institutions. The early data collected had a slice thickness of $1.0 \mathrm{~mm}$ over the acetabulum, $5.0 \mathrm{~mm}$ elsewhere, and an average pixel size of $0.82 \mathrm{~mm} \times 0.82 \mathrm{~mm}$. The later data were collected in helical mode with a slice thickness of 
Table I. Demographic information

\begin{tabular}{|c|c|c|c|c|}
\hline \multirow[b]{3}{*}{ Age (yrs) } & \multicolumn{4}{|c|}{ Mean (range) } \\
\hline & \multicolumn{2}{|c|}{ Female $(n=22)$} & \multicolumn{2}{|c|}{ Male $(n=20)$} \\
\hline & 56.8 & (37 to 79 ) & 54.2 & (38 to 74 ) \\
\hline Height $(\mathrm{cm})$ & 165.4 & (152 to 175 ) & 180.3 & (168 to 190$)$ \\
\hline Weight (kg) & 71.4 & (50 to 100$)$ & 88.0 & (67 to 120$)$ \\
\hline Body mass index $\left(\mathrm{kg} / \mathrm{m}^{2}\right)$ & 26.0 & (20 to 34$)$ & 27.0 & (22 to 35$)$ \\
\hline
\end{tabular}

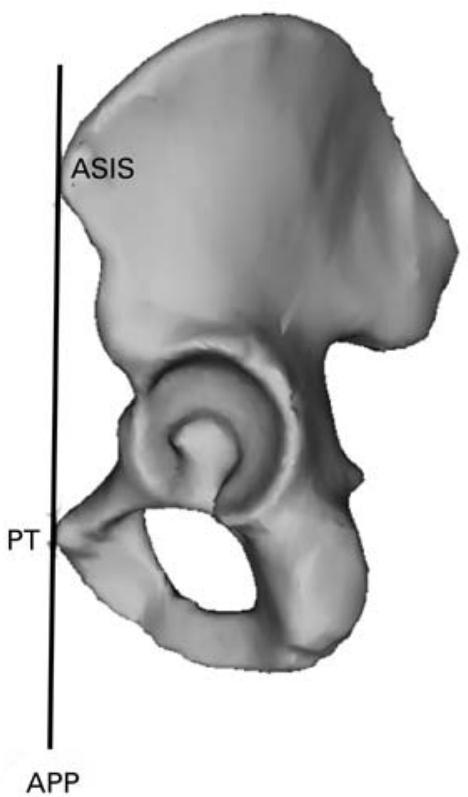

Fig. 1

Left hemipelvis indicating landmarks. The vertical line indicates the anterior pelvic plane (APP) used as a reference plane for the orientation of the pelvis. The most anterior points on the pubic tubercle and anterio superior iliac spine define the APP. Pubic tubercle (PT) indicates the most anterior point on the left pubic tubercle; ASIS indicates the most anterior point on the left anterior superior iliac spine.

$1.25 \mathrm{~mm}$ and average pixel size of $0.79 \mathrm{~mm} \times 0.79 \mathrm{~mm}$. Volumetric CT data provided the reference for the navigation and hence the 3D surface models for the current study. The criteria for inclusion were that the contralateral hip from the THR had been evaluated by the surgeon as healthy on the basis of plain radiographs, no osteophytes were present, and that the quality of the CT scan was high. In addition, the normal hip must not have been distorted by previous surgery, injury, or dysplasia. From the statistical atlas study, a population of high-quality $3 \mathrm{D}$ mesh surfaces from 20 males and 22 females was available for analysis. The patient population spanned a wide range in weight, height and body mass index (Table I). There were 25 left hemipelves and 17 right. The indication for the THR had been degenerative joint disease in all patients. The patients comprised 40 Caucasians and two Afro-Caribbeans.
For each patient, the most anterior point on the anterior superior iliac spine and the midpoint of the most anterior points on the pubic tubercles were extracted from the CT volume (Fig. 1). These landmarks were used to define the anterior pelvic plane, which defines an unambiguous anatomic pelvic co-ordinate system used in navigated THR. ${ }^{12}$

The 3D surface models of the hemipelves were defined as meshes of connected triangles built from vertices segmented from the CT data volume. The lunate surface and rim of the acetabulum were identified on each hemipelvis by selecting the corresponding vertices on that 3D surface model (Fig. 2). Vertices on the acetabular rim were picked by two methods and analysed separately. In the first method, all vertices circumnavigating the acetabular opening but excluding the fossa were picked. In this case, the acetabular orientation was based on the full anatomical opening. In the second method, points restricted to the superior and posterior portions of the rim were picked. This represented rim points that were easily accessible within a surgical opening. One author (PEM) performed the point selection on all of the 3D surface models. A sphere was fitted to the vertices of the lunate surface of the acetabulum and a plane was fitted to vertices along the rim. The parameters for the spheres and planes were determined by minimising the sum of the square of the distances between the vertices and the corresponding geometric shape. The radius of the sphere defined the size of the acetabulum. The abduction and version of the acetabulum were defined by the normal to the rim plane with respect to the anterior pelvic plane.

The depth of the acetabulum, $d$, was defined as the distance between the deepest part of the acetabulum relative to the rim plane (Fig. 3). This depth is of interest because of its influence on the range of movement of the femoral component and acetabular cover. The depth was then normalised to remove the effect of the difference in size between males and females. It was found, on average, that the female acetabular radius is approximately $10 \%$ smaller than that of the male. ${ }^{13}$ The normalised depth, $d_{n}$, is defined as the ratio of the depth to the radius of the fitted sphere $(R)$ :

$$
d_{\mathrm{n}}=d / R
$$

As an alternative but equivalent parameterisation of normalised depth, we calculated the angle $\theta$ subtended by the intersection of the rim plane with the acetabulum:

$$
\theta=2 \cos ^{-1}[(R-d) / R]
$$

These two equivalent parameters allow us to examine the variation in acetabular depth separately from variations in radius.

Statistical analysis. The differences in the values of abduction, version, radius and acetabular depth between males and females were tested for statistical significance using the two-sample Student's $t$-test, assuming unequal variance. The difference in the values of abduction and version from 

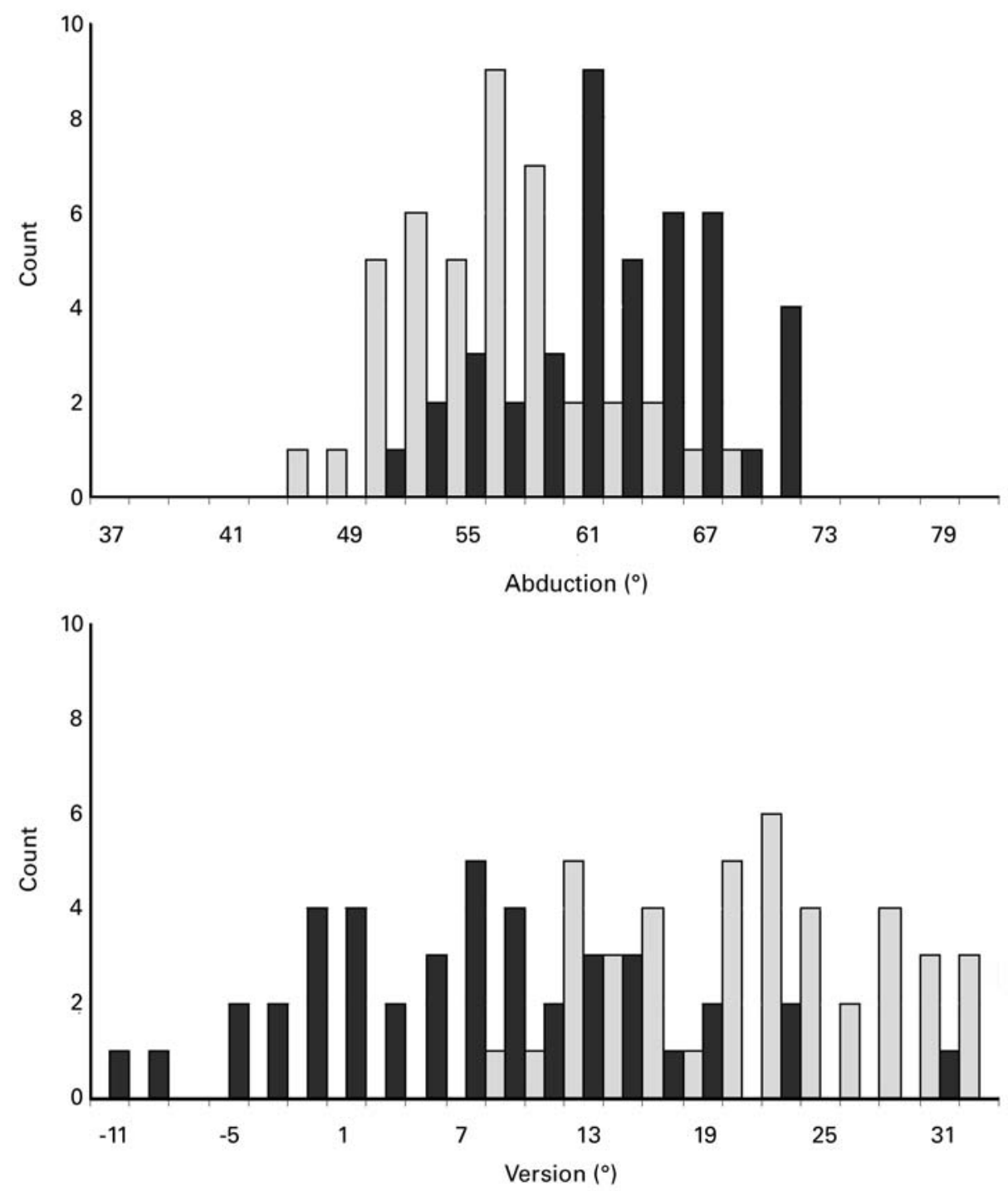

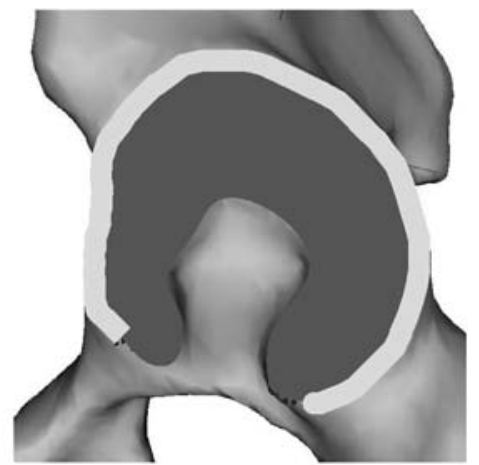

Points selected along full rim

Lunate (wall) surface

Points selected along superior and posterior portion of rim

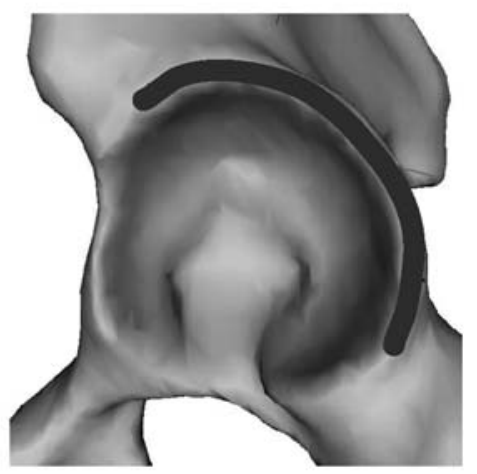

Fig. 2

Distribution of abduction and version based on rim point selection. On the right, the location of vertices used in the least squares matching for the planes of the rim and sphere are indicated. Vertices selected on the lunate surface are in the area shown in dark grey. The light grey curve on the upper pelvis identifies the full rim; light grey bars on the plots on the left indicate the corresponding abduction and version values for planes matched to the full rim co-ordinates. The black curve on the lower pelvis identifies the superior/posterior portions of the acetabular rim; similarly, black bars indicate abduction and version values for planes matched to the points on the superior/posterior rim region.

those corresponding to the predicted safe zone for acetabular component placement were also tested for statistical significance using the same statistical test. Differences were considered significant when $\mathrm{p}<0.05$.

\section{Results}

On average, 310 vertices defined the lunate surface and 84 defined the rim. The standard deviation (SD) of the fit of a sphere to each lunate surface was $0.71 \mathrm{~mm}$ (SD 0.16). This represents the mean of the distances between the fitted sphere and the corresponding points on the lunate surface for all the vertices of the 3D surface models. The SD of the fit of a plane to the vertices along the entire rim was $2.43 \mathrm{~mm}$ (SD 0.67), indicating large variations in rim topography. The SD of the fit of a plane to the vertices limited to the posterior and superior regions of the rim was $0.82 \mathrm{~mm}$ (SD 0.30).

The female acetabulum was orientated with a mean of $57.1^{\circ}$ abduction $\left(50.7^{\circ}\right.$ to $\left.66.8^{\circ}\right)$ and a mean of $24.1^{\circ}$ version $\left(14.0^{\circ}\right.$ to $\left.33.3^{\circ}\right)$. The male acetabulum was orientated with a mean of $55.5^{\circ}$ abduction $\left(47.7^{\circ}\right.$ to $\left.65.9^{\circ}\right)$ and a mean $19.3^{\circ}$ version $\left(8.5^{\circ}\right.$ to $\left.32.3^{\circ}\right)$. There were no retroverted ace- 


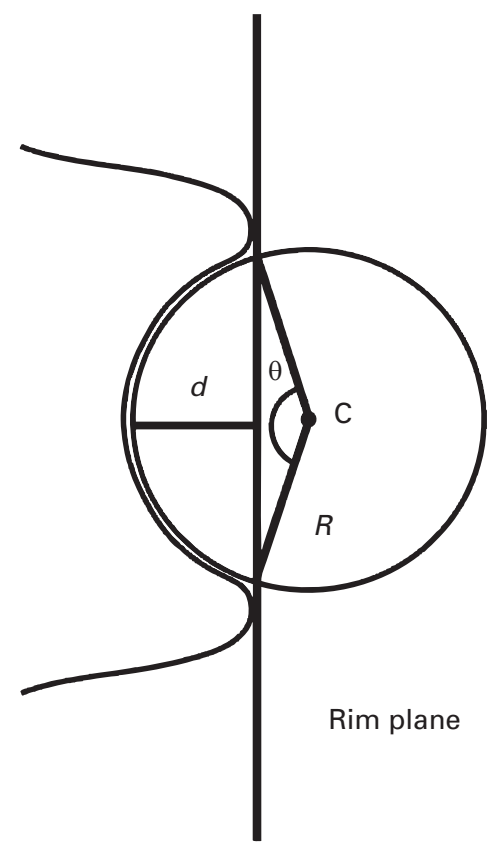

Fig. 3

Depth of the acetabulum is defined as the distance, $d$, between the deepest point in the acetabulum and the plane of the rim. C is the centre and $R$ is the radius of the sphere fitted to the acetabulum. $\theta$ is the angle subtended by the plane of the rim. The normalised depth $(d / R)$ and subtended angle $(\theta)$ allow examination of the variation in acetabular depth separately from variations in size (i.e. variations in radius).

tabula. It was observed that the gender variation for the angle of version was statistically significant $(\mathrm{p}=0.021)$, but the difference for the angle of abduction $(p=0.26)$ was not, signifying that in this study the female acetabulum was more anteverted than that of the male. The range of acetabular abduction was less than that of version in these native acetabula, although both were approximately $20^{\circ}$ or more.

The mean radius of the female acetabulum was $25.0 \mathrm{~mm}$ (21.7 to 30.3); the mean radius of the male acetabulum was $26.7 \mathrm{~mm}$ (24.5 to 28.7). This difference in mean acetabular radius between females and males was found to be statistically significant $(p=0.003)$. We could identify no relationship between body mass index and the size of the acetabulum (regression analysis, females $r=0.001$, males $\mathrm{r}=0.26)$.

The mean depth of the acetabulum was $19.5 \mathrm{~mm} \mathrm{(15.7} \mathrm{to}$ 24.2) for females and $22.7 \mathrm{~mm}$ (17.5 to 28.0) for males. The mean normalised depth for females was $0.79 \mathrm{~mm}(0.56$ to 1.04$)$ and $0.85 \mathrm{~mm}(0.65$ to 0.99$)$ for males. This corresponds to a mean subtended angle of $155.3^{\circ}\left(127.9^{\circ}\right.$ to $\left.184.4^{\circ}\right)$ for females and $162.9^{\circ}\left(138.7^{\circ}\right.$ to $\left.179.1^{\circ}\right)$ for males, suggesting that the female acetabulum is shallower than the male. Nevertheless, the difference in acetabular depth between males and females was not statistically significant when the values were normalised $(\mathrm{p}=0.06$ for normalised depth; $\mathrm{p}=0.058$ for subtended angle).

\section{Discussion}

The surface models used in the study were smoothed to remove the sampling artefacts introduced by layering and pixillation (discretisation) in the CT data volume. The mean maximum distance between the original and the smoothed models was less than $2.82 \mathrm{~mm}$ (SD 0.27). The means of the root mean square and mean distances between the original and smoothed models were $0.46 \mathrm{~mm}$ (SD 0.04) and $0.33 \mathrm{~mm}$ (SD 0.04), respectively. The largest distances between models occurred at the boundaries of the thickest slices, but these points were not within the acetabulum. Although the differences between the smoothed and the original models may appear large, they reflect the fact that the smoothed models were averaging the surface across the CT slice and pixel length. Within the acetabulum, the CT samples were approximately $1 \mathrm{~mm}$ in width, length and height, whereas the average error was less than half that. The 3D surface models were therefore reasonable surfaces from which to measure acetabular properties.

The limitations of this study relate to the assumption that the native parameters of the acetabulum could be determined from the fit of a sphere and a plane to points selected on a 3D surface model of the hemipelvis. Other geometric shapes, such as an ellipsoid, may provide better templates for acetabular parameters. The most significant variation was due to the number and distribution of the points selected for the geometric fits. In order to address this, results from points selected along the entire rim (excluding the fossa) were compared with those selected on the superior and posterior portions of the rim. The study was also limited by the relatively small number of patients. The fact that no retroverted acetabula were observed in our data may be attributable to the small number of patients or to the possible relationship between retroverted acetabula and osteoarthritis, as osteoarthritic acetabula were excluded from the data population.

The radiological safe zones of Lewinnek et $\mathrm{al}^{8}$ have been converted ${ }^{14}$ to anatomical abduction $\left(30.4^{\circ}\right.$ to $\left.54.4^{\circ}\right)$ and version $\left(6.5^{\circ}\right.$ to $\left.43.0^{\circ}\right)$, and are plotted alongside our CT results in Figure 4. Most of the abduction values of the native acetabula measured in this study were greater than the range of abduction values defining the safe zone in the anatomical reference frame, but the range of values for version corresponded to the range for the safe zone. This suggests that the abduction of the native anatomy may not be a good guide for safe placement of the acetabular component.

In order to assess whether the native acetabulum provides a good guide for placement of the prosthetic acetabular component, we tested the hypothesis that the mean radiological inclination and anteversion of the acetabular component safe zone is identical to the same radiological parameters in the native acetabulum. The abduction and version of the native 


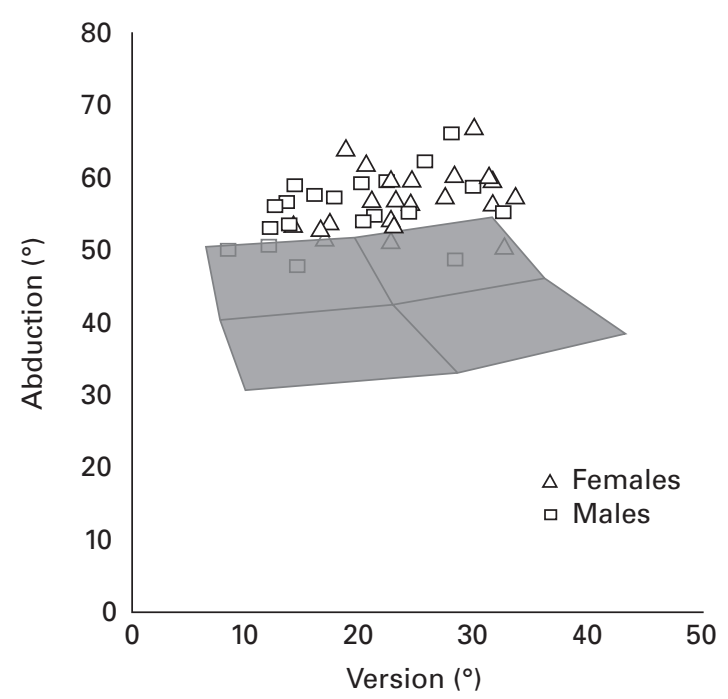

Fig. 4

Anatomical acetabular orientation. The measured native orientation of the acetabulum plotted as a function of gender in the anatomical reference frame. Lewinnek et al's ${ }^{8}$ safe zone is indicated by the grey region.

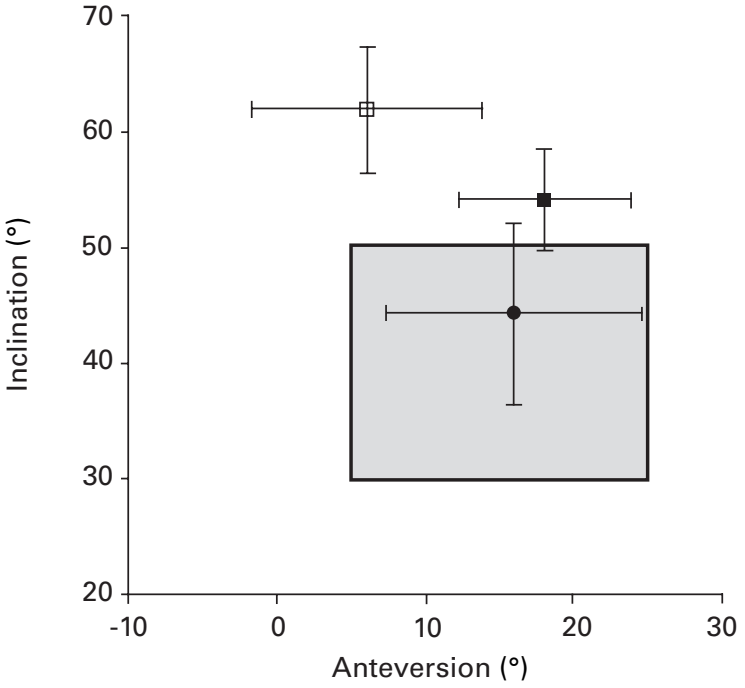

Fig. 5

Average acetabular orientation. Diagram of Lewinnek et al's ${ }^{8}$ safe zone (shaded area) of acetabular component orientation compared with the mean abduction and version from this study converted to the radiological reference frame. The filled square represents results from vertices selected along the full rim; the open square represents results from vertices limited to the superior and posterior portions of the rim. The filled circle is an estimate of the average normal (not dislocated) orientation from Lewinnek et al.

Table II. Summary of hypothesis testing of radiological orientations*

\begin{tabular}{|c|c|c|c|c|c|c|c|}
\hline \multirow[b]{2}{*}{ Description } & \multicolumn{4}{|c|}{ Inclination $(\theta)$ (lateral opening $\left({ }^{\circ}\right)$} & \multicolumn{3}{|c|}{ Anteversion $(\alpha)\left({ }^{\circ}\right)$} \\
\hline & Number & Mean & SD & p-value & $\mu$ & $\sigma$ & p-value \\
\hline Lewinnek et $\mathrm{al}^{8}$ (normal) & 101 & 44.2 & 7.8 & - & 16.0 & 8.6 & - \\
\hline Full rim & 42 & 54.2 & 4.3 & $<0.0001$ & 18.1 & 5.8 & 0.091 \\
\hline Superior and posterior rims & 42 & 61.9 & 5.4 & $<0.0001$ & 6.1 & 7.7 & $<0.0001$ \\
\hline
\end{tabular}

acetabula were determined from the fit of the plane of the rim in the anatomical reference frame and converted to the radiological values of inclination and anteversion (Fig. 4). The mean orientations are plotted against the safe zone in Figure 5. However, the mean inclination values were quite different $(\mathrm{p}<0.01$; Table II), but the mean anteversions could not be distinguished on the basis of these data $(\mathrm{p}=0.091)$. Nevertheless, this demonstrates that the orientation of the native acetabulum as defined by its abduction and version is not a good guide for the safe placement of the acetabular component. Furthermore, the power of the analysis exceeds $99.99 \%$, indicating that there were sufficient data upon which to reach this conclusion.
This comparison with Lewinnek's afe zone may not be ideal, as Rittmeister and Callitsis ${ }^{7}$ analysed the outcomes of 500 THRs and found that dislocation was not more frequent in patients outside the safe zone.

The methodology assumed that a plane fit to the rim of the acetabulum was a reasonable measurement of the orientation of the acetabulum, but the rim comprises three bones (ilium, ischium and pubis) creating an irregular margin. This was highlighted in the SD of $2.43 \mathrm{~mm}$ (SD 0.67) in the fit of the plane of the rim. Selection of points along the rim greatly affects the fit of the plane, and hence the abduction and version. When the vertices were selected primarily on the superior and posterior portions of the rim instead of 
the full rim, the SD of the fit was reduced to $0.82 \mathrm{~mm}$ (SD 0.30 ) and the orientation of the acetabulums was skewed to larger abduction and smaller version values (Fig. 2). The mean abduction became $62.9^{\circ}\left(51.9^{\circ}\right.$ to $\left.71.9^{\circ}\right)$ for females and $61.8^{\circ}\left(52.5^{\circ}\right.$ to $\left.71.2^{\circ}\right)$ for males. The mean angle of version became $10.3^{\circ}\left(-2.0^{\circ}\right.$ to $\left.30.5^{\circ}\right)$ for females and $3.4^{\circ}$ $\left(-0.3^{\circ}\right.$ to $\left.18.1^{\circ}\right)$ for males. The mean subtended angle was $175.0^{\circ}\left(142.8^{\circ}\right.$ to $\left.211.8^{\circ}\right)$ for females and $184.8^{\circ}\left(169.9^{\circ}\right.$ to $207.5^{\circ}$ ) for males. This large shift and range in values raises the question of what is being used as the visual cue to identify acetabular orientation during surgery. Further, the large range in observed orientation of native acetabula may not be well matched by a single targeted mean value for acetabular component placement.

Converting the mean abduction and version angles based on the vertices along the superior and posterior portions of the rim to radiological anteversion and inclination, and comparing them with the mean orientations of the safe zone, revealed statistically significant differences between the two populations ( $\mathrm{p}<0.01$, Table II), and showed that the values lay outside Lewinnek et al's ${ }^{8}$ safe zone (Fig. 5).

As the presence of osteophytes might alter the measurement of native acetabular orientation, arthritic acetabula were excluded from this series. During THR surgeons are advised to remove osteophytes before using the native acetabulum for orientation. Femoral orientation is outside the scope of this study, but surgeons need to bear in mind the importance of the combined anteversion of the femoral and acetabular components. ${ }^{9}$

Stem et $\mathrm{al}^{15}$ suggested that the acetabular anteversion in males was $2.7^{\circ}$ less than that in females. Our results support these findings, but a post hoc power analysis of the version data was $58 \%$, indicating that more patients are required for us to be confident of our findings. For a SD of $5^{\circ}, 45$ male and 45 female measurements would be required to detect a $3^{\circ}$ difference in version between males and females with a power of $80 \%$.

We found that the acetabular radius was gender dependent. The sexual dimorphism was $7 \%$, with the mean radius of the male acetabulum being larger than that of the female. The power for the radius data was $90 \%$, indicating that there was a sufficient number of patients to observe a difference of $1.8 \mathrm{~mm}$ in radius between males and females.

Acetabular depth is important in restoring normal hip mechanics and establishing a good range of movement. It is considered a contributing factor in component dislocation. The range through which the femoral head can move within the acetabular component is related to the subtended angle (normalised depth). By itself, the measured depth gives no indication of the range of movement: the context of the acetabular radius is required. Comparing the subtended angle (normalised depth) of the acetabulum between males and females, we found that female patients had less deep acetabula than males, which produced less cover of the femoral head for the females, although the difference was not statistically significant $(\mathrm{p}=0.06)$.

Based on the mean abduction and version angles measured in this study, positioning on the acetabular component according to native anatomy was not consistent with Lewinnek's safe zone. In particular, the mean abduction angle was larger than recommended by the safe zone theory. To adhere to the safe zone angle, the acetabular component should be approximately $10^{\circ}$ less abducted than the native acetabulum. However, the anteversion of the acetabular component can be more reliably matched to the native anatomy.

No benefits in any form have been received or will be received from a commercial party related directly or indirectly to the subject of this article.

\section{Supplementary Material}

$\because$ A further opinion by Mr C Busch is available with the electronic version of this article on our website at www.jbjs.org.uk

\section{References}

1. McCollum DE, Gray WJ. Dislocation after total hip arthroplasty: causes and prevention. Clin Orthop 1990;261:159-70.

2. Cobb TK, Morrey BF, Ilstrup DM. The elevated-rim acetabular liner in total hip arthroplasty: relationship to postoperative dislocation. J Bone Joint Surg [Am] 1996;78-A:80-6.

3. Mahomed NN, Barrett JA, Katz JN, et al. Rates and outcomes of primary and revision total hip replacement in the United States Medicare Population. J Bone Joint Surg [Am] 2003;85-A:27-32.

4. Phillips CB, Barrett JA, Losina E, et al. Incidence rates of dislocation, pulmonary embolism, and deep infection during the first six months after elective total hip replacement. J Bone Joint Surg [Am] 2003;85-A:20-6.

5. Jolles BM, Zangger $\mathbf{P}$, Leyvraz PF. Factors predisposing to dislocation after primary total hip arthroplasty: a multivariate analysis. J Arthroplasty 2002;17:282-8.

6. Kennedy JG, Rogers WB, Soffe KE, et al. Effect of acetabular component orientation on recurrent dislocation, pelvic osteolysis, polyethylene wear, and component migration. J Arthroplasty 1998;13:530-4.

7. Rittmeister M, Callitsis C. Factors influencing cup orientation in 500 consecutive total hip replacements. Clin Orthop 2006;445:192-6.

8. Lewinnek GE, Lewis JL, Tarr R, Compere CL, Zimmerman JR. Dislocations after total hip-replacement arthroplasties. J Bone Joint Surg [Am] 1978;60-A:217-20.

9. Barsoum WK, Patterson RW, Higuera C, et al. A computer model of the position of the combined component on the prevention of impingement in total hip replacement. J Bone Joint Surg [Br] 2007;89-B:839-45.

10. Archbold HAP, Mockford B, Molby D, et al. The transverse acetabular ligament: an aid to orientation of the acetabular component during primary total hip replacement: a preliminary study of 100 cases investigating post-operative stability. J Bone Joint Surg [Br] 2006;88-B:883-6.

11. Murtha PE, Wu C, Mor AB, Jaramaz B. The influence of sex in the construction of a statistical atlas of the hemi-pelvis [abstract]. J Biomech 2006;39:207.

12. Nikou C, Jaramaz B, DiGioia AM, Levison TJ. Description of anatomic coordinate systems and rationale for use in an image-guided total hip replacement system. In: Delp SL, DiGioia AM, Jaramaz, B, eds. Medical image computing and computerassisted intervention - MICCAI 2000. Springer LNCS 1935: Springer, 2000:1188-94.

13. Hauser DL, Fox JC, Sukin D, Mudge B, Coutts RD. Anatomic variation of structural properties of periacetabular bone as a function of age: a quantitative computed tomography study. J Arthroplasty 1997;12:804-11.

14. Murray DW. The definition and measurement of acetabular orientation. J Bone Joint Surg [Br] 1993;75-B:228-32

15. Stem ES, O'Connor MI, Kransdorf MJ, Crook J. Computed tomography analysis of acetabular anteversion and abduction. Skeletal Radiol 2006:35:385-9. 